Research Paper

\title{
Relationship of genetic variant distributions of WW domain-containing oxidoreductase gene with uterine cervical cancer
}

\author{
Yu-Hsiang Lin",\#, Yi-Hsuan Hsiao,2,3,\#, Wen-Jun Wu11,4, Shun-Fa Yang1,4, Chun-Fang Hsu1, Yu-Ting Kang1, \\ Po-Hui Wang $1,2,5, \bowtie$
}

1. Institute of Medicine, Chung Shan Medical University, Taichung, Taiwan

2. School of Medicine, Chung Shan Medical University, Taichung, Taiwan

3. Department of Obstetrics and Gynecology, Changhua Christian Hospital, Changhua, Taiwan

4. Department of Medical Research, Chung Shan Medical University Hospital, Taichung, Taiwan

5. Department of Obstetrics and Gynecology, Chung Shan Medical University Hospital, Taichung, Taiwan

\#These authors contributed equally to the work.

$\triangle$ Corresponding author: Po-Hui Wang, MD, PhD, Institute of Medicine, Chung Shan Medical University, 110, Section 1, Chien-Kuo North Road, Taichung, 40201, Taiwan. Tel.: 886-4-24739595 ext. 21721; Fax: 884-4-24738493; E-mail: ginhow84921344@yahoo.com.tw

(c) Ivyspring International Publisher. This is an open access article distributed under the terms of the Creative Commons Attribution (CC BY-NC) license (https://creativecommons.org/licenses/by-nc/4.0/). See http://ivyspring.com/terms for full terms and conditions.

Received: 2018.02.14; Accepted: 2018.05.31; Published: 2018.06.14

\begin{abstract}
To our knowledge, no study investigates the association of genetic variant distributions of WW domain-containing oxidoreductase (WWOX) gene with development of invasive cancer, clinicopathologic variables and patient survival in uterine cervical cancer for Taiwanese women. We therefore conducted this study to explore the clinical involvements of WWOX single nucleotide polymorphisms (SNPs) in cervical cancer. One hundred and thirty-one patients with cervical invasive cancer and 93 patients with precancerous lesions as well as 316 control women were consecutively enrolled. The genotypic frequencies of WWOX genetic variants rs73569323, rs383362, rs 11545028, rs 3764340 and rs 12918952 were determined by real-time polymerase chain reaction. The results revealed that only WWOX SNP rs 3764340 was associated between patients with cervical invasive cancer and normal controls among 5 WWOX genetic variants. Cervical cancer patients with genotypes GA/AA in WWOX SNP rs 12918952 were associated with parametrium invasion and pelvic lymph node metastasis. Univariate analysis found that WWOX SNPs rs73569323 and rs 11545028 were associated with patient survival, whereas multivariate analysis revealed CT/TT in rs11545028 was the only genetic variant, which could predict better overall survival, among 5 WWOX SNPs in Taiwan. In conclusion, Taiwanese women with CG/GG in WWOX SNP rs3764340 are susceptible to cervical invasive cancer. Cervical cancer patients with GA/AA in rs 12918952 tend to have more risk to develop parametrium invasion and pelvic lymph node metastasis. Among 5 WWOX SNPs, rsl1545028 is the only genetic variant associated with patient survival, in which CT/TT could predict better overall survival in Taiwanese women.
\end{abstract}

Key words: WW domain-containing oxidoreductase, genetic variants, invasive cancer of uterine cervix, clinicopathologic variables, overall survival

\section{Introduction}

The human WW domain-containing oxidoreductase (WWOX) gene was initially identified by Bednarek et al. and recognized to have two $\mathrm{N}$-terminal WW domains and a short-chain dehydrogenase/reductase central domain in 2000 [1]. It spans the second most active common fragile site
(FRA16D), which is located on chromosome 16q23.-24.1 [2]. The WWOX was considered as a tumor suppressor gene [2-5]. It has been reported that WWOX expression is lost or downregulated in many cancers because of genomic disruption, such as breast $[6,7]$, lung [8] and ovarian cancers [9]. In addition, 
overexpression of WWOX was reported to inhibit the metastasis of human osteosarcoma [10].

When the shared sequence of a gene presents a different single nucleotide between the individuals of a species, single nucleotide polymorphism (SNP) develops [11]. Genetic variant is probably involved in the development and occurrence of certain diseases such as cancers. Genetic polymorphisms may affect the promoter activity and the expression of a gene [11]. It has been reported that SNP may exert a modifying on gene expression and is associated with the risk of breast and ovarian carcinogenesis [12]. Xie et al. found that G>T in WWOX SNP rs383362 is related to an elevated risk of developing chronic obstructive pulmonary disease in a $\mathrm{T}$ allele-number dependent-manner [13]. Furthermore, WWOX genetic variants were reported to be correlated with cancer susceptibility and prognosis [14-17].

The 2013 annual cancer registry report showed that uterine cervical cancer was the second common type of gynecological cancer in Taiwan. It was the third leading cancer in Taiwanese women in 2013. Carcinogenesis of uterine cervix is a multistep process and is known to display a continuum of neoplastic transition from cervical intraepithelial 1 (CIN 1, mild dysplasia; low-grade CIN) to CIN 2 (moderate dysplasia) and CIN3 (severe dysplasia and carcinoma in situ; CIN 2 and CIN 3 regarded as high-grade CIN), then to invasive cancer histologically [18]. Cytologic counterparts of CIN1 as well as CIN2 and 3 correspond to low-grade squamous intraepithelial lesions (LSIL) and high-grade squamous intraepithelial lesions (HSIL), respectively [19]. Moreover, approximately $10 \%$ of LSIL and about $20 \%-30 \%$ of HSIL may progress to invasive cancer of uterine cervix $[20,21]$.

To date, no study associates genetic variant distributions of WWOX with the development of uterine cervical cancer. Also, no study investigates the clinical implication of WWOX SNPs in cervical cancer. Therefore, we conduct this study to explore the relationships among WWOX genetic polymorphism, cervical carcinogenesis, clinicopathologic characteristics and patient survival in Taiwanese women.

\section{Materials and Methods}

\section{Subjects}

Five hundred and forty women, including 131 with invasive cancer, and 93 with precancerous lesions of the uterine cervix, as well as 316 normal controls, were consecutively enrolled into this study. The ages of the women with cervical invasive cancer, precancerous lesions, and normal controls were $55.7 \pm$ 12.6, $43.2 \pm 12.1$ and $44.0 \pm 10.2$, respectively. These normal control groups had neither self-reported history of cancer of any sites. The stages of one hundred and thirty-one women with cervical invasive cancer were assigned according to the 2009 International Federation of Gynecology and Obstetrics Classification. They received routine treatment protocols at the Department of Obstetrics and Gynecology in Chung Shan Medical University Hospital, Taiwan, from August 1993 to August 2014. Ninety-three patients with precancerous lesions received cervical punch biopsy under colposcopy, large loop excision of the transformation zone, total abdominal hysterectomy or total vaginal hysterectomy. The diagnosis of all patients with cervical invasive cancer or precancerous lesions was verified based on the pathologic report before treatment began. All study subjects were Taiwanese women who resided in central Taiwan. The study was approved by the Chung Shan Medical University Hospital Institutional Review Board (CSMUH IRB: CS14014). Written informed consent was obtained from every woman.

\section{Blood samples collection and genomic DNA extraction}

All blood samples were obtained from subjects who participated in this study and placed into Vacutainer tubes containing EDTA and immediately stored at $4^{\circ} \mathrm{C}$. DNA was extracted from white blood cells using the QIAamp DNA blood mini kits (Qiagen, Valencia, California) as previous described [22] . DNA was used as the template in polymerase chain reactions (PCRs).

\section{Selection of genetic variants of WW domain-containing oxidoreductase gene}

In this study, 5 WWOX genetic polymorphisms were selected based on the International HapMap Project data and their potential involvement in the various cancer types [15, 16, 23, 24]. These genetic polymorphisms included WWOX SNPs rs73569323 [exon 8, mRNA position $1683 \mathrm{C}>\mathrm{T}$; 3' untranslated region (3'UTR)], rs383362 (exon 8, 1738 G>T; 3'UTR), rs11545028 (exon 1, 362 C>T; 5’UTR), rs3764340 (exon 7, $1210 \mathrm{C}>\mathrm{G}$ ) and rs12918952 (exon 5, $901 \mathrm{G}>\mathrm{A}$ ). Genotypes of WWOX genetic variants rs73569323 (C_25761998_10), rs383362 (C_2395473_20), rs11545 028 (C_2813530_10), rs3764340 (C_25654217_20) and rs12918952 (C_57888_20) were determined by ABI StepOne Real-Time PCR System (Applied Biosystems, Foster City, CA, USA), and analyzed with SDS vers. 3.0 software, as described previously [24].

\section{Statistical analysis}

ANOVA was applied to compare the age difference among patients with cervical invasive 
cancer and those with precancerous lesions as well as control women, and post hoc analysis was further performed by Scheffe test. Chi-square or Fisher's exact tests were used to examine the relationships among the frequencies of WWOX gene SNPs and alleles and the incidence of cervical neoplasias (including invasive cancer and precancerous lesions). Logistic regression and multinomial logistic regression models were used to analyze multiple comparisons of genotypes of the WWOX gene polymorphisms before and after controlling for age between the patients with cervical neoplasias and the control women as well as among the patients with invasive cancer or precancerous lesions and the controls. Chi-square or Fisher's exact tests were also used to associate the various clinicopathologic parameters with WWOX genetic variants. When the follow-up period was included into survival analysis, the patients were recruited for overall survival between primary surgery and death or the end of the study (December 4 , 2017) using the Kaplan-Meier model for univariate analysis. The curve differences of overall survival in patients with different WWOX gene polymorphisms were assessed by long-rank test. A Cox proportional hazard model with forward stepwise approach was used to evaluate the effects of WWOX SNPs on the overall survival after adjusting for various clinicopathologic characters in multivariate analysis relative to survival time. A $p$ value of less than 0.05 was considered to indicate statistical significance. Odds ratios (ORs) and adjusted odds ratios (AORs, controlling for age) and their 95\% confidence intervals (CIs) were calculated. Hazard ratio (HR) and 95\% CI were also calculated. SPSS software version 22.0 and WinPepi Software version 10.0. were used for statistical analysis.

\section{Results}

There was significant difference for age distribution between patients with cervical neoplasia and normal control women (50.2 \pm 13.8 vs. $44.0 \pm 10.2$, $p<0.001)$. The age difference displayed statistically significant between patients with cervical invasive cancer and those with precancerous lesions (55.7 \pm 12.6 vs. $43.2 \pm 12.1, p<0.001)$ as well as between those with cervical invasive cancer and control women (55.7 \pm 12.6 vs. $44.0 \pm 10.2, p<0.001$ ). But no significant difference of age distribution existed between those with precancerous lesions and control women (43.2 \pm 12.1 vs. $44.0 \pm 10.2, p=0.803$ ).

The genotypic frequencies of WWOX SNP rs3764340 conformed to Hardy- Weinberg equilibrium in the normal controls $\left[p=0.720, \chi^{2}\right.$ value: $0.13<5.99$, degree of freedom=2]. Distributions of other WWOX SNPs rs73569323, rs11545028 and rs12918952 satisfied the equilibrium ( $p=0.561, \chi^{2}$ value: 0.33 ; $p=0.940, \chi^{2}$ value: $0.01 ; p=0.584, \chi^{2}$ value: 0.32 , respectively).

\section{Relationship of WWOX genetic variant distributions with uterine cervical neoplasias}

The genotypic frequencies of WWOX SNPs in the Taiwanese women with cervical neoplasias and the control women are summarized in Table 1 . There were no significantly different distributions of WWOX SNPs, rs73569323, rs383362, rs11545028, rs3764340 and rs12918952 between patients with cervical neoplasias and normal controls. Even after controlling for age, no significant differences still existed for these SNPs between patients with cervical neoplasias and normal controls.

\section{Association of WWOX genetic polymorphisms with uterine cervical carcinogenesis}

When the cervical neoplasia group was classified into subgroups of invasive cancer and precancerous lesions, only WWOX SNP rs3764340 was found to be differently distributed between patients with cervical invasive cancer and normal controls among 5 WWOX genetic variants (Table 2). Even controlling for age, women with CG/GG in WWOX rs3764340 still had more risk of developing invasive cancer, using CC as a reference (AOR: 1.91, 95\% CI: 1.13-3.23, $p=0.016$; Table 2).

However, there were no significantly different genotypes for this SNP between patients with cervical precancerous lesions and normal controls.

\section{Association among allele distributions among WWOX SNPs, cervical invasive cancer and precancerous lesions and normal controls}

Regarding the allelic frequencies of the 5 WWOX gene polymorphisms in the 540 specimens collected, the allele G in SNP rs3764340 increased the risk to develop cervical invasive cancer after controlling for age using $\mathrm{C}$ as a comparison reference (AOR: 1.96, 95\% CI: 1.10-3.49, $p=002$; Table 3). However, no different allelic frequencies were noted for other WWOX SNPs.

\section{The correlation of WWOX genetic polymorphisms with clinicopathologic characteristics in the cervical cancer patients}

In relating the various clinicopathologic parameters to WWOX genetic variants, there was no association of WWOX gene polymorphism rs3764340 with clinicopathologic parameters (Table 4). However, patients with genotypes GA/AA in WWOX SNP rs12918952 tended to have more risk of parametrium invasion $(p=0.028, \quad \mathrm{OR}=4.25, \quad 95 \%$ 
$\mathrm{CI}=0.96-19.14)$ and pelvic lymph node metastasis $(p=0.034, \mathrm{OR}=4.02,95 \% \mathrm{CI}=0.92-10.03)$ than those with genotype GG (Table 4). Moreover, other WWOX SNPs rs73569323, rs383362, rs11545028 were not associated with clinicopathologic variables.

\section{Univariate analysis Kaplan-Meier curves model for the survival in patients with cervical cancer based on WWOX genetic polymorphisms}

When we associated the WWOX genetic polymorphisms with patient survival, we found that the overall survival in cervical patients with CT/TT in rs73569323 were inferior to that in patients with wild homozygote CC $(p=0.022$; HR: 5.342, 95\% CI: 1.065-26.789; Table 5). In contrast, patients with
CT/TT in rs11545028 had better overall survival than those with wild homozygote CC $(p<0.001$; HR: 0.158, $95 \%: 0.056-0.450)$. However, we could not demonstrate cervical cancer patients with genotypes GT/TT in rs383362, CG/GG in rs3764340 or GA/AA in rs12918952 had better overall survival than those with GG, CC or GG using Kaplan-Meier curves ( $p=0.338$, $p=0.512$ or $p=0.510$ ) Moreover, patients with wild homozygous/heterozygous genotypes in all 5 WWOX polymorphisms were not better than those with mutant homozygous genotype ( $p$ unavailable, $p$ unavailable, $p=0.182, p$ unavailable, or $p$ unavailable for rs73569323, rs383362, rs11545028, rs3764340 or rs12918952 separately).

Table 1. Genetic variant distributions of WW domain-containing oxidoreductase gene in Taiwanese patients with neoplasias of the uterine cervix and normal controls

\begin{tabular}{|c|c|c|c|c|c|c|}
\hline Variables & Normal controls $(n=316)$ & Cervical neoplasiasa $(\mathrm{n}=224)$ & $p$ values & Odds ratios (95\% CIs) & Adjusted $p^{\mathrm{b}}$ & Adjusted odds ratios $(95 \% \mathrm{CIs})^{\mathrm{b}}$ \\
\hline \multicolumn{7}{|l|}{ rs73569323 } \\
\hline $\mathrm{CC}^{\mathrm{c}}$ & 296 & 214 & 0.351 & 1.00 & & 1.00 \\
\hline CT & 20 & 10 & & $0.69(0.32-1.51)$ & 0.572 & $0.79(0.35-1.80)$ \\
\hline TT & 0 & 0 & & - & - & - \\
\hline $\mathrm{CC}^{\mathrm{c}}$ & 296 & 214 & 0.351 & 1.00 & 0.572 & 1.00 \\
\hline $\mathrm{CT} / \mathrm{TT}$ & 20 & 10 & & $0.69(0.32-1.51)$ & & $0.79(0.35-1.80)$ \\
\hline $\mathrm{CC} / \mathrm{CT}^{\mathrm{c}}$ & 316 & 224 & u.a. & 1.00 & u.a. & 1.00 \\
\hline TT & 0 & 0 & & u.a. & & u.a. \\
\hline \multicolumn{7}{|l|}{ rs383362 } \\
\hline $\mathrm{GG}^{\mathrm{c}}$ & 238 & 179 & 0.210 & 1.00 & & 1.00 \\
\hline GT & 78 & 45 & & $0.77(0.51-1.16)$ & 0.236 & $0.77(0.50-1.19)$ \\
\hline TT & 0 & 0 & & u.a. & u.a. & u.a. \\
\hline $\mathrm{GG}^{\mathrm{c}}$ & 238 & 179 & 0.210 & 1.00 & 0.236 & 1.00 \\
\hline GT/TT & 78 & 45 & & $0.77(0.51-1.17)$ & & $0.77(0.50-1.19)$ \\
\hline GG/GTc & 316 & 224 & - & 1.00 & u.a. & 1.00 \\
\hline TT & 0 & 0 & & u.a. & & u.a. \\
\hline \multicolumn{7}{|l|}{ rs11545028 } \\
\hline $\mathrm{CC}^{\mathrm{c}}$ & 178 & 133 & 0.832 & 1.00 & & 1.00 \\
\hline CT & 118 & 79 & & $0.90(0.63-1.30)$ & 0.547 & $0.89(0.61-1.31)$ \\
\hline TT & 20 & 13 & & $0.88(0.42-1.83)$ & 0.389 & $0.71(0.33-1.55)$ \\
\hline $\mathrm{CC}^{\mathrm{c}}$ & 178 & 132 & 0.547 & 1.00 & 0.421 & 1.00 \\
\hline CT/TT & 138 & 92 & & $0.90(0.64-1.27)$ & & $0.86(0.60-1.24)$ \\
\hline $\mathrm{CC} / \mathrm{CT}^{\mathrm{c}}$ & 296 & 211 & 0.802 & 1.00 & 0.446 & 1.00 \\
\hline TT & 20 & 13 & & $0.91(0.44-1.87$ & & $0.74(0.34-1.60)$ \\
\hline \multicolumn{7}{|l|}{ rs3764340 } \\
\hline $\mathrm{CC}^{\mathrm{c}}$ & 275 & 182 & 0.160 & 1.00 & & 1.00 \\
\hline CG & 40 & 40 & & $1.51(0.94-2.43)$ & 0.108 & $1.52(0.91-2.52)$ \\
\hline GG & 1 & 2 & & $3.02(0.27-33.57)$ & 0.377 & $3.00(0.26-34.28)$ \\
\hline $\mathrm{CC}^{\mathrm{c}}$ & 275 & 182 & 0067 & 1.00 & 0.082 & 1.00 \\
\hline CG/GG & 41 & 42 & & $1.55(0.97-2.48)$ & & $1.56(0.95-2.57)$ \\
\hline $\mathrm{CC} / \mathrm{CG}^{\mathrm{c}}$ & 315 & 222 & 0.573 & 1.00 & 0.404 & 1.00 \\
\hline GG & 1 & 2 & & $2.84(0.26-31.49)$ & & $2.82(0.25-32.18)$ \\
\hline \multicolumn{7}{|l|}{ rs12918952 } \\
\hline$G^{c}$ & 276 & 197 & 0.925 & 1.00 & & 1.00 \\
\hline GA & 38 & 25 & & $0.92(0.54-1.58)$ & 0.854 & $0.95(0.54-1.67)$ \\
\hline AA & 2 & 2 & & $1.40(0.20-10.03)$ & 0.369 & $2.49(0.34-18.20)$ \\
\hline $\mathrm{GG}^{c}$ & 276 & 197 & 0.834 & 1.00 & 0.979 & 1.00 \\
\hline GA/AA & 40 & 27 & & $0.95(0.56-1.59)$ & & $1.01(0.58-1.74)$ \\
\hline GG/GAc & 314 & 222 & 1.000 & 1.00 & 0.365 & 1.00 \\
\hline $\mathrm{AA}$ & 2 & 2 & & $1.41(0.20-10.12)$ & & $2.51(0.34-18.30)$ \\
\hline
\end{tabular}

Statistical analysis: logistic regression model or chi-square or Fisher's exact tests.

aCervical neoplasias included precancerous lesions and invasive cancer of the uterine cervix.

bThe adjusted $p$ values as well as adjusted odds ratios and their $95 \%$ confident intervals were estimated by logistic regression model after controlling for age.

cUsed as a reference for comparison to evaluate the odds ratios of other genotypes. $95 \%$ CIs, 95\% confidence intervals; u.a., unavailable. 
Table 2. Genetic variant distributions of WW domain-containing oxidoreductase gene in Taiwanese patients with uterine cervical invasive cancer or precancerous lesions and normal controls.

\begin{tabular}{|c|c|c|c|c|c|c|}
\hline Variables & $\begin{array}{l}\text { Normal controls } \\
(\mathrm{n}=316)\end{array}$ & $\begin{array}{l}\text { Precancerous lesions } \\
(\mathrm{n}=93)\end{array}$ & $\begin{array}{l}\text { Invasive cancer } \\
(\mathrm{n}=131)\end{array}$ & $p$ values & $\begin{array}{l}\text { Adjusted } p \text { values \& AORs }(95 \% \\
\text { CIs) }{ }^{\mathrm{b}}\end{array}$ & $\begin{array}{l}\text { Adjusted } p \text { values \& AORs (95\% } \\
\text { CIs) })^{c}\end{array}$ \\
\hline \multicolumn{7}{|c|}{ rs73569323 } \\
\hline $\mathrm{CC}^{\mathrm{d}}$ & 296 & 89 & 125 & 0.645 & 1.00 & 1.00 \\
\hline $\mathrm{CT}$ & 20 & 4 & 6 & & $\begin{array}{l}0.467 \\
0.67(0.22-2.00)\end{array}$ & $\begin{array}{l}0.474 \\
0.71(0.28-1.81)\end{array}$ \\
\hline TT & 0 & 0 & 0 & & $\begin{array}{l}\text { u.a. } \\
\text { u.a. }\end{array}$ & $\begin{array}{l}\text { u.a. } \\
\text { u.a. }\end{array}$ \\
\hline $\mathrm{CC}^{\mathrm{d}}$ & 296 & 89 & 125 & 0.645 & 1.00 & 1.00 \\
\hline $\mathrm{CT} / \mathrm{TT}$ & 20 & 4 & 6 & & $\begin{array}{l}0.467 \\
0.67(0.22-2.00)\end{array}$ & $\begin{array}{l}0.474 \\
0.71(0.28-1.81)\end{array}$ \\
\hline $\mathrm{CC} / \mathrm{CT}^{\mathrm{d}}$ & 316 & 93 & 131 & u.a & 1.00 & 1.00 \\
\hline $\mathrm{TT}$ & 0 & 0 & 0 & & $\begin{array}{l}\text { u.a } \\
\text { u.a }\end{array}$ & $\begin{array}{l}\text { u.a } \\
\text { u.a }\end{array}$ \\
\hline \multicolumn{7}{|l|}{ rs383362 } \\
\hline $\mathrm{GG}^{\mathrm{d}}$ & 238 & 74 & 105 & 0.453 & 1.00 & 1.00 \\
\hline GT & 78 & 19 & 26 & & $\begin{array}{l}0.397 \\
0.78(0.45-1.38)\end{array}$ & $\begin{array}{l}0.272 \\
0.76(0.46-1.25)\end{array}$ \\
\hline TT & 0 & 0 & 0 & & $\begin{array}{l}\text { u.a } \\
\text { u.a }\end{array}$ & $\begin{array}{l}\text { u.a } \\
\text { u.a }\end{array}$ \\
\hline $\mathrm{GG}^{\mathrm{d}}$ & 238 & 74 & 105 & 0.453 & 1.00 & 1.00 \\
\hline GT/TT & 78 & 19 & 26 & & $\begin{array}{l}0.397 \\
0.78(0.45-1.38)\end{array}$ & $\begin{array}{l}0.272 \\
0.76(0.46-1.25)\end{array}$ \\
\hline GG/GTd & 316 & 93 & 131 & u.a & 1.00 & 1.00 \\
\hline TT & 0 & 0 & 0 & & $\begin{array}{l}\text { u.a } \\
\text { u.a }\end{array}$ & $\begin{array}{l}\text { u.a } \\
\text { u.a }\end{array}$ \\
\hline \multicolumn{7}{|c|}{ rs11545028 } \\
\hline $\mathrm{CC}^{\mathrm{d}}$ & 178 & 54 & 78 & 0.973 & 1.00 & 1.00 \\
\hline $\mathrm{CT}$ & 118 & 34 & 45 & & $\begin{array}{l}0.836 \\
0.95(0.58-1.55)\end{array}$ & $\begin{array}{l}0.531 \\
0.87(0.56-1.34)\end{array}$ \\
\hline TT & 20 & 5 & $\underline{8}$ & & $\begin{array}{l}0.712 \\
0.82(0.30-2.30)\end{array}$ & $\begin{array}{l}0.836 \\
0.91(0.39-2.16)\end{array}$ \\
\hline $\mathrm{CC}^{\mathrm{d}}$ & 178 & 54 & 78 & 0.814 & 1.00 & 1.00 \\
\hline $\mathrm{CT} / \mathrm{TT}$ & 138 & 39 & 53 & & $\begin{array}{l}0.767 \\
0.93(0.58-1.49)\end{array}$ & $\begin{array}{l}0.532 \\
0.88(0.58-1.33)\end{array}$ \\
\hline $\mathrm{CC} / \mathrm{CT}^{\mathrm{d}}$ & 296 & 88 & 123 & 0.945 & 1.00 & 1.00 \\
\hline TT & 20 & 5 & 8 & & $\begin{array}{l}0.736 \\
0.84(0.31-2.31)\end{array}$ & $\begin{array}{l}0.930 \\
0.96(0.41-2.24)\end{array}$ \\
\hline \multicolumn{7}{|l|}{ rs3764340 } \\
\hline $\mathrm{CC}^{\mathrm{d}}$ & 275 & 80 & 102 & 0.083 & 1.00 & 1.00 \\
\hline CG & 40 & 12 & 28 & & $\begin{array}{l}0.930 \\
1.03(0.52-2.06)\end{array}$ & $\begin{array}{l}0.02^{\mathrm{a}} \\
1.89(1.11-3.22)\end{array}$ \\
\hline GG & 1 & 1 & 1 & & $\begin{array}{l}0.385 \\
3.44(0.21-55.57)\end{array}$ & $\begin{array}{l}0.485 \\
2.70(0.17-43.51)\end{array}$ \\
\hline $\mathrm{CC}^{\mathrm{d}}$ & 275 & 80 & 102 & $0.046^{\mathrm{a}}$ & 1.00 & 1.00 \\
\hline CG/GG & 41 & 13 & 29 & & $\begin{array}{l}0.802 \\
1.09(0.56-2.13)\end{array}$ & $\begin{array}{l}0.016^{\mathrm{a}} \\
1.91(1.13-3.23)\end{array}$ \\
\hline $\mathrm{CC} / \mathrm{CG}^{\mathrm{d}}$ & 315 & 92 & 130 & 0.373 & 1.00 & 1.00 \\
\hline GG & 1 & 1 & 1 & & $\begin{array}{l}0.386 \\
3.42(0.21-55.27)\end{array}$ & $\begin{array}{l}0.533 \\
2.42(0.15-39.03)\end{array}$ \\
\hline \multicolumn{7}{|c|}{ rs12918952 } \\
\hline $\mathrm{GG}^{\mathrm{d}}$ & 276 & 79 & 118 & 0.429 & 1.00 & 1.00 \\
\hline GA & 38 & 12 & 13 & & $\begin{array}{l}0.782 \\
1.10(0.55-2.21)\end{array}$ & $\begin{array}{l}0.512 \\
0.80(0.41-1.56)\end{array}$ \\
\hline AA & 2 & 2 & 0 & & $\begin{array}{l}0.215 \\
3.49(0.48-25.20)\end{array}$ & $\begin{array}{l}\text { u.a } \\
\text { u.a }\end{array}$ \\
\hline $\mathrm{GG}^{\mathrm{d}}$ & 276 & 79 & 118 & 0.506 & 1.00 & 1.00 \\
\hline GA/AA & 40 & 14 & 13 & & $\begin{array}{l}0.549 \\
1.22(0.63-2.36)\end{array}$ & $\begin{array}{l}0.417 \\
0.76(0.39-1.47)\end{array}$ \\
\hline $\mathrm{GG} / \mathrm{GA}^{\mathrm{d}}$ & 314 & 91 & 131 & 0.185 & 1.00 & 1.00 \\
\hline AA & 2 & 2 & 0 & & $\begin{array}{l}0.219 \\
3.45(0.48-24.84)\end{array}$ & $\begin{array}{l}\text { u.a } \\
\text { u.a }\end{array}$ \\
\hline
\end{tabular}

Statistical analysis: multinomial logistic regression or chi-square or Fisher's exact tests, ${ }^{\mathrm{a}} p<0.05$.

bAdjusted $p$ values and adjusted odds ratios with their 95\% CIs were estimated by multinomial logistic regression models after controlling for age between patients with cervical precancerous lesions and control women.

cAdjusted $p$ values and adjusted odds ratios with their 95\% CIs were estimated by multinomial logistic regression models after controlling for age between patients with cervical invasive cancer and control women.

dUsed as a reference for comparison to evaluate the odds ratios of other genotypes.

AORs, adjusted odds ratios; 95\% CIs, 95\% confidence intervals; u.a., unavailable. 
Table 3. Allele distribution of WW domain-containing oxidoreductase gene in Taiwanese patients with uterine cervical invasive cancer or precancerous lesions and normal controls.

\begin{tabular}{|c|c|c|c|c|c|c|}
\hline Variables & $\begin{array}{l}\text { Normal controls } \\
(\mathrm{n}=316)\end{array}$ & $\begin{array}{l}\text { Precancerous lesions } \\
(\mathrm{n}=93)\end{array}$ & $\begin{array}{l}\text { Invasive cancer } \\
(\mathrm{n}=131)\end{array}$ & $p$ values & $\begin{array}{l}\text { Adjusted } p \text { values \& AORs (95\% } \\
\text { CIs) }{ }^{\mathrm{b}}\end{array}$ & $\begin{array}{l}\text { Adjusted } p \text { values \& AORs (95\% } \\
\text { CIs) }\end{array}$ \\
\hline rs73569323 & & & & 0.653 & 0.457 & 1.000 \\
\hline $\mathrm{C}^{\mathrm{d}}$ & 612 & 182 & 256 & & 1.00 & 1.00 \\
\hline $\mathrm{T}$ & 20 & 4 & 6 & & $0.66(0.22-1.96)$ & $1.00(0.35-2.86)$ \\
\hline rs383362 & & & & 0.502 & 0.442 & 0.386 \\
\hline $\mathrm{G}^{\mathrm{d}}$ & 554 & 167 & 236 & & 1.00 & 1.00 \\
\hline $\mathrm{T}$ & 78 & 19 & 26 & & $0.81(0.48-1.38)$ & $0.79(0.46-1.35 f)$ \\
\hline rs11545028 & & & & 0.837 & 0.603 & 0.339 \\
\hline $\mathrm{Cd}^{\mathrm{d}}$ & 474 & 142 & 201 & & 1.00 & 1.00 \\
\hline $\mathrm{T}$ & 158 & 44 & 61 & & $0.90(0.61-1.33)$ & $0.83(0.56-1.22)$ \\
\hline rs3764340 & & & & 0.054 & 0.694 & $0.02^{\mathrm{a}}$ \\
\hline $\mathrm{Cd}^{\mathrm{d}}$ & 590 & 172 & 232 & & 1.00 & 1.00 \\
\hline G & 42 & 14 & 30 & & $1.14(0.61-2.13)$ & 1.96 (1.10-3.49) \\
\hline rs12918952 & 590 & 170 & 249 & 0.307 & 0.365 & 0.599 \\
\hline $\mathrm{G}^{\mathrm{d}}$ & 42 & 16 & 13 & & 1.00 & 1.00 \\
\hline A & & & & & $0.82(0.40-1.69)$ & $0.82(0.40-1.69)$ \\
\hline
\end{tabular}

Statistical analysis: multinomial logistic regression or chi-square test, ap $<0.05$. Adjusted $p$ values and adjusted odds ratios with their $95 \%$ CIs were estimated by multinomial logistic regression models after controlling for age. a $<<0.05$.

${ }^{\mathrm{b}}$ Adjusted $p$ values and adjusted odds ratios with their $95 \%$ CIs were estimated by multinomial logistic regression models after controlling for age between patients with cervical precancerous lesions and control women.

cAdjusted $p$ values and adjusted odds ratio with their 95\% CIs were estimated by multinomial logistic regression models after controlling for age between patients with cervical invasive cancer and control women.

dUsed as a reference to evaluate the odds ratios.

Abbreviations: AORs, adjusted odds ratios; $95 \%$ CIs, $95 \%$ confidence intervals.

Table 4. Association of genotypic distribution of WW domain-containing oxidoreductase genetic polymorphisms rs 3764340 and rs 12918952 with clinicopathologic variables of the patients with invasive cancer of uterine cervix.

\begin{tabular}{|c|c|c|c|c|c|c|c|c|}
\hline \multirow[t]{2}{*}{ Variablesa } & \multicolumn{2}{|c|}{ rs3764340 } & \multirow[t]{2}{*}{$p$ value } & \multirow[t]{2}{*}{ ORs (95\% CIs) } & \multicolumn{2}{|c|}{ rs12918952 } & \multirow[t]{2}{*}{$p$ value } & \multirow[t]{2}{*}{ ORs (95\% CIs) } \\
\hline & $\mathrm{CC}^{\mathrm{b}}$ & CG/GG & & & $\mathrm{GG}^{\mathrm{b}}$ & GA/AA & & \\
\hline Clinical stage & & & 0.894 & & & & 0.053 & \\
\hline stage $I^{b}$ & 55 & 17 & & 1.00 & 68 & 4 & & 1.00 \\
\hline$\geq$ stage II & 31 & 9 & & $0.94(0.33-2.55)$ & 33 & 7 & & $3.61(0.84-17.80)$ \\
\hline Pathologic type & & & 0.758 & & & & 0.211 & \\
\hline squamous cell carcinomab & 72 & 23 & & 1.00 & 84 & 11 & & 1.00 \\
\hline adenocarcinoma & 14 & 3 & & $0.67(0.11-2.73)$ & 17 & 0 & & $0.00(0.00-2.21)$ \\
\hline Cell grading & & & 0.456 & & & & 1.000 & \\
\hline well $(\text { grade } 1)^{\mathrm{b}}$ & 18 & 7 & & 1.00 & 23 & 2 & & 1.00 \\
\hline moderate \& poor (grades $2 / 3$ ) & 68 & 18 & & $0.68(0.23-2.24)$ & 77 & 9 & & $1.34(0.25-13.62)$ \\
\hline Stromal invasion depth & & & 0.197 & & & & 0.527 & \\
\hline$\leq 10 \mathrm{~mm}^{\mathrm{b}}$ & 46 & 17 & & 1.00 & 58 & 5 & & 1.00 \\
\hline$>10 \mathrm{~mm}$ & 40 & 8 & & $0.54(0.18-1.50)$ & 42 & 6 & & $1.66(0.39-7.32)$ \\
\hline Tumor diameterb & & & 0.072 & & & & 0.189 & \\
\hline$\leq 4 \mathrm{~cm}$ & 52 & 20 & & 1.00 & 67 & 5 & & 1.00 \\
\hline$>4 \mathrm{~cm}$ & 34 & 5 & & $0.38(0.10-1.19)$ & 33 & 6 & & $2.44(0.57-10.80)$ \\
\hline Parametrium & & & 0.228 & & & & $0.028^{c}$ & \\
\hline no invasion ${ }^{b}$ & 62 & 21 & & 1.00 & 78 & 5 & & 1.00 \\
\hline invasion & 24 & 4 & & $0.49(0.11-1.69)$ & 22 & 6 & & $4.25(0.96-19.14)$ \\
\hline Vagina & & & 0.711 & & & & 0.692 & \\
\hline no invasion ${ }^{b}$ & 65 & 20 & & 1.00 & 78 & 7 & & 1.00 \\
\hline invasion & 29 & 5 & & $0.56(0.15-1.75)$ & 22 & 3 & & $1.52(0.23-7.34)$ \\
\hline Pelvic lymph node & & & 0.428 & & & & $0.034^{c}$ & \\
\hline no metastasis ${ }^{b}$ & 62 & 20 & & 1.00 & 77 & 5 & & 1.00 \\
\hline metastasis & 24 & 5 & & $0.65(0.17-2.06)$ & 23 & 6 & & $4.02(0.92-10.03)$ \\
\hline
\end{tabular}

Statistical analyses: chi-square or Fisher's exact tests, $c p<0.05$

aSome clinicopathological data could not be obtained from the patients with cervical invasive cancer due to incomplete medical charts or records.

bAs a reference. ORs, odds ratios; $95 \%$ CIs, $95 \%$ confidence intervals.

\section{Multivariate analysis Cox proportional hazard model for the survival in patients with cervical cancer based on WWOX genetic polymorphisms}

In multivariate analysis, we found rs11545028 is the only independent predictor of cervical cancer patient survival among 5 WWOX genetic polymorphisms after adjusting for clinicopathologic variables. Patients with genotypes CT/TT in rs11545028 had better prognosis than those with CC ( $p=0.002$; HR: 0.167, 95\% CI 0.054-0.518; Table 5). However, there were no associations among rs73569323 as well as other WWOX SNPs and patient survival in Cox proportional hazard model analysis. 
Table 5. Univariate and multivariate analyses for the association of WW domain-containing oxidoreductase (WWOX) genetic polymorphisms with survival of the patients with uterine cervical cancer.

\begin{tabular}{|c|c|c|}
\hline \multirow[t]{2}{*}{ Variables } & \multicolumn{2}{|c|}{ Overall survival } \\
\hline & $p$ value & $\mathrm{HR} \& 95 \% \mathrm{CI}^{c}$ \\
\hline \multicolumn{3}{|l|}{ Univariate analysis ${ }^{a}$} \\
\hline rs73569323 CT/TT vs CC ${ }^{b}$ & 0.022 & $5.342(1.065-26.789)$ \\
\hline rs11545028 CT/TT vs CCb & $<0.001$ & $0.158(0.056-0.450)$ \\
\hline \multicolumn{3}{|l|}{ Multivariate analysisa } \\
\hline rs $11545028 \mathrm{CT} / \mathrm{TT}$ vs $\mathrm{CC}^{\mathrm{b}}$ & 0.002 & $0.167(0.054-0.518)$ \\
\hline
\end{tabular}

Statistical analyses: aunivariate analysis: Kaplan-Meier curve model; amultivariate analysis: Cox proportional hazard model, forward stepwise approach

bAs a comparison reference

cHR, hazard ratio and 95\% CI, 95\% confidence interval for WWOX genetic polymorphisms after adjusting for clinicopathologic variables, compared to their respective controls.

\section{Discussion}

Although no different distribution in 5 WWOX genetic variants was found between patients with cervical neoplasia and normal controls in Taiwanese women, the only genotypic difference was demonstrated in WWOX SNP rs3764340 after cervical neoplasias were subdivided into subgroups of invasive cancer and precancerous lesions in this study. This different genotype was not found between patients with precancerous and normal controls. The effect of genotypes CG/GG on invasive cancer might be masked by the precancerous lesion cases. In this study, Taiwanese women with CG or CG/GG were related to the development of cervical invasive cancer using CC as a reference in genetic variant distribution of WWOX SNP rs3764340. It may be attributable to the low sample size in Taiwanese women for the failed demonstration of the mutant homozygous GG effect (CC as a comparison reference) in WWOX SNP rs3764340 on the development of cervical invasive cancer.

$\mathrm{Qu}$ et al. showed that WWOX expression is reduced in human cervical cancer and cervical cancer cell lines [25]. WWOX expression has been reported to be involved in the regulation of cell cycle, apoptosis, angiogenesis and tumorigenesis [26-28]. Reconstruction of WWOX in HeLa cervical cancer cells inhibited cell proliferation and triggered apoptosis, while knockdown of WWOX in SiHa cells enhanced cell proliferation and inhibited apoptosis. Giarnieri et al. found that the WWOX expression is reduced in high rates of cervical cancer and inferred that alterations of WWOX gene may lead to cervical carcinogenesis [29]. To our knowledge, no study associated WWOX SNPs with susceptibility of cervical cancer. However, we had the novel finding that Allele G in WWOX SNP rs3764340 is associated with the development of cervical invasive cancer even after controlling for age and Taiwanese women with CG/GG are susceptible to cervical invasive cancer, using $\mathrm{CC}$ as a reference. In agreement with our finding, Cancemi et al. demonstrated that risk of thyroid cancer is increased in individuals carrying the G-allele in WWOX polymorphism Pro282Ala (rs3764340, 1210 C>G) [30]. Although no other variant distribution of WWOX gene was found between patients with cervical invasive cancer and normal controls in our study, Cheng et al. identified that subjects who carry TT or CT/TT of WWOX polymorphism rs11545028 are more susceptible to oral cancer than those who carry wild homozygote CC [23]. In contrast, Lee et al. found a significant association of WWOX SNP rs73569323 with decreased risk of hepatocellular carcinoma (HCC) [24].

WWOX polymorphic variants have been reported to be associated with with aggressiveness and poor prognosis in a variety of cancers, such as lung cancer, pancreatic cancer, and esophageal squamous cell carcinoma [15, 31, 32]. Furthermore, we related WWOX SNP rs3764340 to clinicopathologic variables of cervical cancer. However, we could not demonstrate an association between rs3764340 and clinicopathologic factors. Our results revealed that WWOX SNP rs12918952 was the only genetic variant that was associated with clinicopathologic factors of cervical cancer among 5 WWOX genetic polymorphisms. Patients with GA/AA in rs12918952 tended to have more risk of parametrium invasion and pelvic lymph node metastasis. WWOX SNP rs12918952 is located in the exon 5 of WWOX gene and heterozygous/mutant homozygous genotypes GA/AA may result in amino acid change (nonsynonymous SNP, dbSNP allele GCA>ACA, mRNA position 901, protein position 179, alanine>threonine) $[11,23]$ and then loss of the tumor suppression function of WWOX gene [2]. It is reasonable that rs12918952 SNP is correlated with the progression of cervical cancer. In agreement with our findings, the A allele in WWOX SNP rs12918952 was reported to indicate a higher aggressive phenotypic risk of vascular invasion [24].

Although our study could not demonstrate WWOX SNP rs12918952 was associated with cervical cancer survival, we found rs73569323 and rs11545028 are associated with patient survival. Univariate analysis showed that cervical cancer patients with genotypes CT/TT in rs73569323 carried a risk of poor overall survival than those with wild homozygote CC. In contrast, heterozygote $\mathrm{CT} /$ mutant homozygote CC in WWOX SNP rs11545028 indicated better patient survival as compared to wild homozygote CC. Abnormal WWOX expression was reported to be associated with a poor prognosis in breast cancer [33]. WWOX has been reported to be lost in $30 \%$ of ovarian cancer and was associated with disease progression 
and poor prognosis [34]. It was conferred that genotypic variant of WWOX SNP rs11545028 influences WWOX gene expression, which was significantly associated with tumor phenotypes and subsequently with tumor development and aggressiveness [23]. Płuciennik et al. found that high level expression of WWOX was associated with improved disease free survival [35]. In our multivariate analysis, CT/TT in rs 11545028 was the only genetic variant, which was associated with cervical cancer patient survival among 5 WWOX SNPs and it could predict better overall survival.

Patients with at least one T allele in WWOX SNP rs11545028 were identified to have a significantly smaller tumor size, decreased levels of alpha-fetoprotein and alanine aminotransferase in hepatocellular carcinoma [24]. In general, patients with these characters have more chance to carry better prognosis.

In conclusion, our study reveals that Taiwanese women with CG/GG in WWOX SNP rs3764340 are susceptible to cervical invasive cancer. Cervical cancer patients with GA/AA in rs12918952 tend to have more risk to develop parametrium invasion and pelvic lymph node metastasis. Although univariate analysis indicates WWOX genetic variants rs73569323 and rs11545028 are associated with patient survival, multivariate analysis reveals CT/TT in rs11545028 is the only genetic variant, which could predict better overall survival among 5 WWOX SNPs in Taiwanese women.

\section{Acknowledgments}

This study was supported by research grants from Ministry of Science and Technology (MOST 105-2314-B-040-016-MY2) and Chung Shan Medical University Hospital (CSH-2018-D-004).

\section{Competing Interests}

The authors have declared that no competing interest exists.

\section{References}

[1] Bednarek AK, Laflin KJ, Daniel RL, Liao Q, Hawkins KA and Aldaz CM. WWOX, a novel WW domain-containing protein mapping to human chromosome 16q23.3-24.1, a region frequently affected in breast cancer. Cancer Res 2000; 60: 2140-2145.

[2] Paige AJ, Taylor KJ, Taylor C, Hillier SG, Farrington S, Scott D, Porteous DJ, Smyth JF, Gabra H and Watson JE. WWOX: a candidate tumor suppressor gene involved in multiple tumor types. Proc Natl Acad Sci U S A 2001; 98: 11417-11422.

[3] Del Mare S, Salah Z and Aqeilan RI. WWOX: its genomics, partners, and functions. J Cell Biochem 2009; 108: 737-745.

[4] Lo JY, Chou YT, Lai FJ and Hsu LJ. Regulation of cell signaling and apoptosis by tumor suppressor WWOX. Exp Biol Med (Maywood) 2015; 240: 383-391.

[5] Hsu LJ, Chiang MF, Sze CI, Su WP, Yap YV, Lee IT, Kuo HL and Chang NS. HYAL-2-WWOX-SMAD4 Signaling in Cell Death and Anticancer Response. Front Cell Dev Biol 2016; 4: 141.

[6] Guler G, Uner A, Guler N, Han SY, Iliopoulos D, Hauck WW, McCue P and Huebner K. The fragile genes FHIT and WWOX are inactivated coordinately in invasive breast carcinoma. Cancer 2004; 100: 1605-1614.
[7] Nunez MI, Ludes-Meyers J, Abba MC, Kil H, Abbey NW, Page RE, Sahin A, Klein-Szanto AJ and Aldaz CM. Frequent loss of WWOX expression in breast cancer: correlation with estrogen receptor status. Breast Cancer Res Treat 2005; 89: 99-105.

[8] Iliopoulos D, Guler G, Han SY, Johnston D, Druck T, McCorkell KA, Palazzo J, McCue PA, Baffa R and Huebner K. Fragile genes as biomarkers: epigenetic control of WWOX and FHIT in lung, breast and bladder cancer. Oncogene 2005; 24: 1625-1633.

[9] Gourley C, Paige AJW, Taylor KJ, Scott D, Francis NJ, Rush R, Aldaz CM, Smyth JF and Gabra H. WWOX mRNA expression profile in epithelial ovarian cancer supports the role of WWOX variant 1 as a tumour suppressor, although the role of variant 4 remains unclear. Int J Oncol 2005; 26: 1681-1689.

[10] Del Mare S and Aqeilan RI. Tumor Suppressor WWOX inhibits osteosarcoma metastasis by modulating RUNX2 function. Sci Rep 2015; 5: 12959.

[11] Shastry BS. SNPs: impact on gene function and phenotype. Methods Mol Biol 2009; 578: 3-22.

[12] Yarden RI, Friedman E, Metsuyanim S, Olender T, Ben-Asher E and Papa MZ. MDM2 SNP309 accelerates breast and ovarian carcinogenesis in BRCA1 and BRCA2 carriers of Jewish-Ashkenazi descent. Breast Cancer Res Treat 2008; 111: 497-504.

[13] Xie C, Chen X, Qiu F, Zhang L, Wu D, Chen J, Yang L and Lu J. The role of WWOX polymorphisms on COPD susceptibility and pulmonary function traits in Chinese: a case-control study and family-based analysis. Sci Rep 2016; 6: 21716.

[14] Yang L, Liu B, Huang B, Deng J, Li H, Yu B, Qiu F, Cheng M, Wang H, Yang R, Yang $X$, Zhou $Y$ and $\mathrm{Lu} \mathrm{J}$. A functional copy number variation in the WWOX gene is associated with lung cancer risk in Chinese. Hum Mol Genet 2013; 22: 1886-1894.

[15] Schirmer MA, Luske CM, Roppel S, Schaudinn A, Zimmer C, Pfluger R, Haubrock M, Rapp J, Gungor C, Bockhorn M, Hackert T, Hank T, Strobel O, Werner J, Izbicki JR, Johnsen SA, Gaedcke J, Brockmoller J and Ghadimi BM. Relevance of $\mathrm{Sp}$ Binding Site Polymorphism in WWOX for Treatment Outcome in Pancreatic Cancer. J Natl Cancer Inst 2016; 108:

[16] Paige AJ, Zucknick M, Janczar S, Paul J, Mein CA, Taylor KJ, Stewart M, Gourley C, Richardson S, Perren T, Ganesan TS, Smyth JF, Brown R and Gabra $\mathrm{H}$. WWOX tumour suppressor gene polymorphisms and ovarian cancer pathology and prognosis. Eur J Cancer 2010; 46: 818-825.

[17] Lin WR, Hsu CW, Yeh CS, Chen YC, Chang ML, Liang KH, Lin CC, Chu YD and Yeh CT. Combinations of single nucleotide polymorphisms WWOX-rs13338697, GALNT14-rs9679162 and rs6025211 effectively stratify outcomes of chemotherapy in advanced hepatocellular carcinoma. Asia Pac J Clin Oncol 2018; 14: e54-e63.

[18] Su SC, Hsieh MJ, Yang WE, Chung WH, Reiter RJ and Yang SF. Cancer metastasis: Mechanisms of inhibition by melatonin. J Pineal Res 2017; 62:

[19] Khan MJ, Castle PE, Lorincz AT, Wacholder S, Sherman M, Scott DR, Rush BB, Glass AG and Schiffman M. The elevated 10-year risk of cervical precancer and cancer in women with human papillomavirus (HPV) type 16 or 18 and the possible utility of type-specific HPV testing in clinical practice. J Natl Cancer Inst 2005; 97: 1072-1079.

[20] Bharti AC, Shukla S, Mahata S, Hedau S and Das BC. Anti-human papillomavirus therapeutics: facts \& future. Indian J Med Res 2009; 130: 296-310.

[21] Baak JP, Kruse AJ, Robboy SJ, Janssen EA, van Diermen B and Skaland I. Dynamic behavioural interpretation of cervical intraepithelial neoplasia with molecular biomarkers. J Clin Pathol 2006; 59: 1017-1028.

[22] Su SC, Hsieh MJ, Lin CW, Chuang CY, Liu YF, Yeh CM and Yang SF. Impact of HOTAIR Gene Polymorphism and Environmental Risk on Oral Cancer. J Dent Res 2018; 22034517749451.

[23] Cheng HL, Liu YF, Su CW, Su SC, Chen MK, Yang SF and Lin CW. Functional genetic variant in the Kozak sequence of WW domain-containing oxidoreductase (WWOX) gene is associated with oral cancer risk. Oncotarget 2016; 7: 69384-69396.

[24] Lee HL, Cheng HL, Liu YF, Chou MC, Yang SF and Chou YE. Functional genetic variant of WW domain-containing oxidoreductase (WWOX) gene is associated with hepatocellular carcinoma risk. PLoS One 2017; 12: e0176141.

[25] Qu J, Lu W, Li B, Lu C and Wan X. WWOX induces apoptosis and inhibits proliferation in cervical cancer and cell lines. Int J Mol Med 2013; 31: 1139-1147.

[26] Yan H, Tong J, Lin X, Han Q and Huang H. Effect of the WWOX gene on the regulation of the cell cycle and apoptosis in human ovarian cancer stem cells. Mol Med Rep 2015; 12: 1783-1788.

[27] Li G, Sun L, Mu Z, Huang Y, Fu C and Hu B. Ectopic WWOX Expression Inhibits Growth of 5637 Bladder Cancer Cell In Vitro and In Vivo. Cell Biochem Biophys 2015; 73: 417-425.

[28] Seabra MAL, Candido EB, Vidigal PVT, Lamaita RM, Rodrigues AN and Silva Filho ALD. Immunohistochemical WWOX Expression and Association with Angiogenesis, p53 Expression, Cell Proliferation and Clinicopathological Parameters in Cervical Cancer. Rev Bras Ginecol Obstet 2018; 40: 79-85.

[29] Giarnieri E, Zanesi N, Bottoni A, Alderisio M, Lukic A, Vecchione A, Ziparo V, Croce $\mathrm{CM}$ and Mancini R. Oncosuppressor proteins of fragile sites are reduced in cervical cancer. Cancer Lett 2010; 289: 40-45.

[30] Cancemi L, Romei C, Bertocchi S, Tarrini G, Spitaleri I, Cipollini M, Landi D, Garritano S, Pellegrini G, Cristaudo A, Pinchera A, Barale R, Elisei R, Landi S and Gemignani F. Evidences that the polymorphism Pro-282-Ala within the 
tumor suppressor gene WWOX is a new risk factor for differentiated thyroid carcinoma. Int J Cancer 2011; 129: 2816-2824.

[31] Huang D, Qiu F, Yang L, Li Y, Cheng M, Wang H, Ma G, Wang Y, Hu M, Ji W,

Zhou Y and $\mathrm{Lu}$ J. The polymorphisms and haplotypes of WWOX gene are associated with the risk of lung cancer in southern and eastern Chinese populations. Mol Carcinog 2013; 52 Suppl 1: E19-27.

[32] Guo W, Wang G, Dong Y, Guo Y, Kuang G and Dong Z. Decreased expression of WWOX in the development of esophageal squamous cell carcinoma. Mol Carcinog 2013; 52: 265-274.

[33] Gothlin Eremo A, Wegman P, Stal O, Nordenskjold B, Fornander T and Wingren $\mathrm{S}$. Wwox expression may predict benefit from adjuvant tamoxifen in randomized breast cancer patients. Oncol Rep 2013; 29: 1467-1474.

[34] Nunez MI, Rosen DG, Ludes-Meyers JH, Abba MC, Kil H, Page R, Klein-Szanto AJ, Godwin AK, Liu J, Mills GB and Aldaz CM. WWOX protein expression varies among ovarian carcinoma histotypes and correlates with less favorable outcome. BMC Cancer 2005; 5: 64.

[35] Pluciennik E, Kusinska R, Potemski P, Kubiak R, Kordek R and Bednarek AK. WWOX--the FRA16D cancer gene: expression correlation with breast cancer progression and prognosis. Eur J Surg Oncol 2006; 32: 153-157. 